\title{
EL ACCESO IRRESTRICTO DE ESTUDIANTES A LAS UNIVERSIDADES ARGENTINAS A TRAVÉS DE LOS DISCURSOS DE LA PRENSA DIARIA (1982-1983)
}

DOI: http://dx.doi.org/10.1590/2236-3459/76889

\author{
Sara González Gómez \\ Universidad de las Islas Baleares, Palma/Islas Baleares, España \\ Guillermo Ramón Ruiz \\ Universidad de Buenos Aires - Conicet, Buenos Aires, Argentina
}

\begin{abstract}
Resumen
Históricamente, los estudiantes han desempeñan un rol primordial en la lucha por la mejora de la universidad. En Argentina, tras la derrota en la Guerra de Las Malvinas (1982), se produjo la reactivación del movimiento estudiantil y con ella la disputa por la consecución del acceso irrestricto a los estudios superiores. En este trabajo se toman tres variables como objeto de estudio - universidad, estudiantes y acceso irrestricto - que se analizan esencialmente a partir del estudio de la prensa diaria (Clarín, La Prensa y La Nación). El objetivo fundamental es poner en evidencia la posición de los estudiantes respecto al tipo de procedimiento para el acceso a la universidad durante la época de la transición a la democracia (considerada aquí entre junio de 1982 y finales del año 1983) y la forma de hacerla visible o efectiva ante las autoridades y, por ende, ante el panorama público, al darse visibilidad de ello en la prensa ${ }^{1}$.

Palabras clave: universidad, estudiantes, ingreso irrestricto, Argentina, prensa.
\end{abstract}

\section{O ACESSO IRRESTRITO DE ESTUDANTES ÀS UNIVERSIDADES ARGENTINAS ATRAVÉS DOS DISCURSOS DA IMPRENSA DIÁRIA (1982-1983)}

\section{Resumo}

Historicamente, os estudantes desempenharam um papel primordial na luta por uma universidade melhor. $\mathrm{Na}$ Argentina, depois da derrota na Guerra das Malvinas (1982), aconteceu a reativação do movimento estudantil e, com ele, a disputa pelo acesso sem restrições aos estudos superiores. Neste trabalho, tomamos três variáveis no nosso objeto de estudo - universidade, estudantes e acesso sem restrições - cuja análise realizase, essencialmente, a partir do estudo da imprensa diária (Clarín, La Prensa y La Nación). O objetivo fundamental é colocar em evidência o posicionamento dos estudantes em relação ao tipo de procedimentos

\footnotetext{
${ }^{1}$ Este trabajo se inscribe en una de las principales líneas de investigación del grupo Ágora de Educación (Universidad de Valladolid) que tiene como objetivo fundamental analizar los procesos de construcción de imaginarios pedagógicos colectivos a través de la prensa diaria en tiempos de cambio político y social en la Europa Mediterránea e Iberoamérica.
} 
para o acesso à universidade durante a época de transição para a democracia (considerada aqui entre junho de 1982 e final do ano de 1983) e a forma de torná-la visível ou efetiva perante as autoridades e o panorama público ao ganhar visibilidade na imprensa.

Palavras-chave: universidade, estudantes, acesso sem restrições, Argentina, imprensa.

\title{
THE UNRESTRICTED ACCESS OF STUDENTS TO THE ARGENTINE UNIVERSITIES THROUGH THE DISCOURSES OF THE DAILY PRESS (1982-1983)
}

\begin{abstract}
This paper focuses on the political positions that the university student movement has taken during the Argentine democratic transition. Moreover, we analyze -through the press- the several conflicts and proposals that the university students have had regarding the university admission policies that were implemented during the 1976-1983 dictatorship last years and during the democratic transition period (1982-1983). After the Malvinas' war end, the student movement started to protest in order to get a different university admission policy: more open, without academic restrictions and with free tuition. Those demands had gotten diverse responses by the military government, which show its weakness and they also illustrate the beginning of the democratic transition period. We take into account three dimensions to analyze this process: the public universities, the university student movement, and the university admission policies. We look at three newspapers (Clarín, La Nación and La Prensa) to study the student protests, their demands, and the policies that the government and the universities has taken during those years regarding the university admission. Keywords: university, students, free access, Argentina, press.
\end{abstract}

\section{L'ACCES ILLIMITE DES ETUDIANTS AUX UNIVERSITES ARGENTINES A TRAVERS LES DISCOURS DE LA PRESSE QUOTIDIENNE (1982-1983)}

\section{Résumé}

Historiquement, les étudiants ont joué un rôle clé dans la lutte pour l'amélioration de l'université. En Argentine, après la défaite dans la guerre des Malouines (1982), il y avait la renaissance du mouvement étudiant et avec elle le différend sur la poursuite de l'accès illimité à l'enseignement supérieur. Dans cet article, nous prenons trois variables comme un objet d'étude - université, étudiants et l'accès sans restriction - qui est essentiellement analysé de l'étude des journaux quotidiens (Clarín, La Prensa et La Nación). L'objectif principal est de mettre la situation des étudiants en ce qui concerne le type de procédure d'accès à l'université au cours de la période de transition vers la démocratie (considéré ici entre Juin 1982 et fin 1983) et comment le faire en preuve visible ou effectif devant les autorités et donc à la scène publique, pour lui donner une visibilité dans la presse

Mots-clés: Université, étudiants, accès sans restriction, Argentine, journaux. 


\section{Introducción}

lo largo de la historia, los estudiantes han jugado un papel fundamental en
la lucha por la consecución de derechos y condiciones democráticas en el
seno de las universidades de todo el mundo. Con sus críticas abiertas contra el orden existente, los movimientos estudiantiles fueron erigiéndose en potentes fuerzas reivindicativas que han merecido la atención de diferentes estudiosos. Tal y como sostiene Carrillo Linares (2015, p. 65), "juventud" y "universidad" constituyen dos variables de análisis de enorme fecundidad para comprender las transformaciones y sus consecuencias, aunque existen otras que completan la explicación, como puedan ser los diversos movimientos sociales (obrero, vecinal, entre otros), los cambios institucionales, las tendencias y relaciones internacionales, el desarrollismo económico, el análisis de los grupos dirigentes, por mencionar algunos. En el devenir histórico todos interactuaron simultáneamente, a diferentes ritmos o escalas y con diversos efectos.

En el caso de la Argentina, durante la última dictadura militar iniciada en 1976, el sistema de cupos para los estudios de grado universitarios se convirtió en una de las formas a partir de las cuales se manifestó el control sobre el acceso a la universidad pública². Ello condicionó el hecho de que las luchas estudiantiles por la democratización se tradujeran en la demanda de ingreso irrestricto o directo, es decir, sin pruebas de admisión. (SIGAL, 2003). Una vez iniciada la fase de transición democrática, tras la caída en la Guerra de Las Malvinas, las demandas estudiantiles por su consecución se acrecentaron. De hecho, poco después, el gobierno del presidente Alfonsín se propuso democratizar las instituciones universitarias e interpelaría especialmente para ello a la juventud estudiantil con el objetivo de construir la democracia en diversos ámbitos. (VOMMARO, 2016, p. 27) ${ }^{3}$. En el terreno universitario, uno de esos ámbitos, y de los primeros en alcanzarse, sería el del acceso irrestricto a las universidades. Históricamente, hablar de ingreso irrestricto a la universidad en Argentina implica historiar una tradición que no es lineal, que tiene sus momentos fundacionales, sus continuidades y discontinuidades. Ligado estrechamente al principio de igualdad de oportunidades educativas, el ingreso irrestricto o ingreso libre ha sido considerado, por muchos especialistas, como uno de los rasgos que le dio a la universidad argentina su carácter abierto y público. (PIERELLA, 2015).

Todo este proceso que nos planteamos abordar se enmarca en un contexto internacional mucho más amplio en el cual es posible identificar dos cuestiones clave: por un lado, la denominada por Huntington (1990) como "tercera ola" de transiciones a la democracia, iniciada en la década de los 70 , que conducirá a un número elevado de países al tránsito de regímenes autoritarios hacia sistemas democráticos, entre ellos Argentina,

\footnotetext{
2 El nivel superior del sistema educativo argentino está constituido por dos sectores muy diferentes: el universitario y el terciario no universitario. En ambos hay instituciones públicas y privadas. La diferencia más importante entre ambos sectores viene dada por el hecho de que en la actualidad el sector terciario en su conjunto depende de los gobiernos de cada una de las 24 jurisdicciones que posee el Estado federal argentino, ya que ha sido completamente descentralizado entre los años 1992 y 1994 . El sector universitario a su vez posee diferentes tipos de instituciones públicas, algunas bajo la órbita del Estado nacional (universidades nacionales e institutos universitarios estatales) y otras que dependen de los Estados provinciales (universidades provinciales). Desde el año 1994, las universidades nacionales tienen autonomía y autarquía reconocida en la Constitución Nacional, o sea, que la autonomía universitaria es un atributo constitucional que debe ser respetado por las leyes que apruebe el Poder Legislativo.

${ }^{3}$ Raúl Alfonsín (1927-2009) fue presidente de Argentina entre el 10 de diciembre de 1983 y el 8 de julio de 1989; su gobierno constituyó el primero democrático luego del período de terrorismo de Estado iniciado en 1976 después del golpe cívico militar que derrocó al gobierno de María Estela Martínez, viuda de Perón.
} 
con todas las implicaciones de cambio y transformación que este tipo de procesos suponen para las distintas esferas política, social, económica, cultural y educativa de cada país (GONZÁLEZ, 2015); por otro lado, el fenómeno global de democratización de las universidades, asociado a la conversión de un modelo educativo elitista dirigido a una minoría en un modelo de universidad masificada, todavía lejos del concepto de universidad de masas, pero al que de forma progresiva comienzan a acceder un mayor número de estudiantes, procedentes de diferentes estratos sociales, que contribuye a la ruptura de las tradicionales coordenadas de estas instituciones de formación superior.

Dicho esto, el objetivo principal de este trabajo es rastrear los discursos que sobre el acceso irrestricto de los estudiantes a las universidades argentinas se emitieron en la prensa diaria de la época, concebida como una parcela de la esfera pública donde los intereses de grupos diversos y las opiniones de individuos varios, con capacidad para influir sobre la opinión pública, confluyen en un mismo soporte. (ARENT, 1993, 1998; HABERMAS, 1994). Durante la época de la transición a la democracia, la prensa se habría convertido en una suerte de espejo público -también de cuestiones relacionadas con la educación, dada su relevancia social-con posibilidades diversas y capacidad para influir, al fin, sobre el imaginario social (TAYLOR, 2006) y la opinión pública. De tal modo que el uso de la prensa ${ }^{4}$ como fuente nos ofrecería una buena oportunidad para rearmar las posturas, pareceres y prioridades de los estudiantes en sus reclamos para alcanzar la consecución de una universidad diferente durante aquella época de transición a la democracia. La orientación del periódico marca el tipo de noticia registrada, la forma en que ha sido contada y los datos que se han incluido $u$ omitido en ella, y todo ello ha sido tenido en cuenta. Mediante el método histórico, y las pertinentes críticas interna y externa de nuestras fuentes, teniendo como referente de trabajo los principios del análisis crítico del discurso (VAN DIJK, 1999), tratamos de rearmar el discurso de esos estudiantes, desde la óptica de los diferentes medios de prensa escrita, ante la reclamación del acceso irrestricto a la universidad.

Para este trabajo se han seleccionado tres periódicos (La Nación, La Prensa y Clarín) y dentro de los mismos todos aquellos editoriales y artículos de opinión editados durante la etapa de transición hacia la democracia, considerado aquí como tal el periodo que va desde el fin de la Guerra de Las Malvinas (junio de 1982) hasta la celebración de las elecciones democráticas en octubre de 1983. Estos tres periódicos se han escogido en función de criterios de mayor difusión y teniendo en cuenta que todos ellos comparten una serie de rasgos comunes entre sí: en primer lugar, fueron fundados por personas que han tenido un peso específico en la sociedad argentina, vinculadas directamente con la política; en segundo término, la dirección de los rotativos ha estado, desde su creación, en herederos de los fundadores, y ello ha ubicado a esas familias entre las más influyentes de Argentina, y de algún modo, en responsables de la construcción de los imaginarios colectivos del país. (HERNÁNDEZ; GONZÁLEZ, 2014, p. 184).

El corpus documental de partida que ha sido manejado para este trabajo lo constituyen un total de 85 recortes de prensa, 26 correspondientes al periódico Clarín, 50 a La Nación y 9 a La Prensa. Estos artículos de opinión se centran en cuestiones

\footnotetext{
${ }^{4}$ Tal y como mencionan Hernández y Ortega (2015), en el caso particular de Argentina, la prensa diaria es un campo de investigación escasamente atendido por los historiadores de la educación, un material original y novedoso con dimensiones, calado y valor específicos aún por determinar.
} 
relacionadas con los estudiantes y la universidad de forma genérica, y abordan temas relacionados especialmente con las elecciones de representantes de estudiantes, el proceso de normalización, las protestas del movimiento estudiantil y la cuestión específica del acceso irrestricto a los estudios universitarios, habiendo sido referenciados al final del texto sólo los manejados y citados a lo largo del trabajo.

\section{El contexto inmediato a la transición democrática}

En el año 1976 se produjo un golpe de Estado en la Argentina que derrocó al gobierno constitucional de María Estela Martínez de Perón e instauró, como órgano supremo del Estado, una Junta de Comandantes con representación de las tres fuerzas armadas (ejército, marina y aviación). Se inició a partir de ese momento el autodenominado como "proceso de reorganización nacional" que cambiaría radicalmente la estructura política, social, económica, cultural y educativa del país y que se caracterizó por la violación sistemática de los derechos humanos en un contexto de terrorismo de Estado.

Dentro del ámbito universitario, el gobierno dictatorial impuso un sistema de regulación caracterizado por el intervencionismo, el control y la vigilancia políticoideológica, la desvinculación de ciencia y universidad y, entre otras cosas, por la redefinición de los planes de estudio y los contenidos de las materias en función de las preferencias del régimen. (KAUFMANN, 2017). Durante ese periodo, las instituciones de educación superior fueron intervenidas, se volvió a la política de cupos y exámenes de ingreso, se estableció un control riguroso en las universidades nacionales que no fueron cerradas y se intentó poner en marcha un plan cuyos principales objetivos eran reducir las dimensiones del sistema, redistribuir la matrícula y canalizar hacia ámbitos extrauniversitarios las actividades de investigación científica. (BUCHBINDER, 2005, p. 209). Se prohibieron todas las actividades políticas e institucionales, así como la existencia de centros de estudiantes y actividades sindicales de los docentes. Profesores y estudiantes fueron cesanteados o bien secuestrados, asesinados y desaparecidos en el marco de un proyecto represivo.

Unos años más tarde, tras una fuerte crisis del gobierno autoritario, se produciría un acontecimiento que vendría a desestabilizar y a dar por iniciado el desarme de la dictadura: la guerra de las Malvinas, también denominada como guerra del Atlántico Sur. Esta contienda enfrentó a la Argentina y el Reino Unido por el control y la soberanía de los archipiélagos de las Malvinas, Georgias del Sur y Sándwich del Sur. A mediados del año 1982, el enfrentamiento se saldó con la derrota argentina y este hecho precipitó la caída de la Junta Militar, que convocó a elecciones democráticas para octubre del año siguiente.

Durante los años 1982 y 1983 se estableció una constante pugna entre continuidades y rupturas y los partidos políticos iniciaron la competencia preelectoral. En lo que concierne al ámbito educativo, todos los partidos comenzaron a plantear estrategias tendentes a la resolución de los problemas unánimemente reconocidos como principales: la deserción y la repetición escolar, el deterioro de la calidad de la educación, la burocratización y la excesiva centralización de las decisiones, los contenidos curriculares. (BRASLAVSKY et al., 1989, p. 45). En las universidades, la derrota militar en las Malvinas y el inicio de la campaña electoral para los comicios de octubre de 1983 provocaron la reanimación de la vida política y las diferentes agrupaciones de estudiantes comenzaron a reorganizarse siguiendo parámetros partidarios. Asimismo, se inauguró un proceso de 
normalización de las universidades para garantizar el funcionamiento de las casas de estudios según sus estatutos ${ }^{5}$, los concursos permitieron el regreso de científicos y académicos cualificados que habían sido apartados durante la dictadura y la investigación científica volvió a recuperar centralidad como función de la universidad. (BUCHBINDER, 2005 , p. 212 y p. 216). Cabe destacar que todas estas iniciativas políticas conllevaron muchos años y esfuerzos políticos e institucionales de parte de todos los actores involucrados. Su consecución plena fue cercenada por las constantes crisis económicas que afectaron el financiamiento educativo desde el retorno a la democracia en 1983 y cuyas consecuencias perviven en la actualidad.

El proceso de normalización universitaria se inició -en el terreno normativo- a través de la promulgación del Decreto 154/83. Este decreto presidencial fue dictado a tres días de haber asumido el gobierno constitucional. En él se dispuso la intervención de las universidades nacionales, la rehabilitación provisional de los estatutos vigentes al 29 de julio de 1966 y otras medidas transitorias, sin llegar a derogar totalmente la Ley № $22.207^{6}$. Seis meses después, el Congreso Nacional sancionó la Ley № 23.068 de 1984, que ratificó aquel Decreto 154/83, con algunas modificaciones, y derogó definitivamente la Ley № 22.207. (RUIZ; CARDINAUX, 2010; CANTINI, 1997).

Entre otras consecuencias, el conflicto de las islas Malvinas posibilitó el renacimiento de la militancia en las universidades como en otros ámbitos de la vida institucional argentina. Pocos días después de la fecha de inicio del conflicto (2 de abril de 1982), la comisión multipartidaria recibió a un grupo de representantes de la Federación Universitaria Argentina (FUA) encabezada por su entonces presidente, Roberto Vázquez. El eje central de los reclamos de los dirigentes era la limitación al ingreso universitario (BUCHBINDER; MARQUINA, 2008, p. 18) y, de hecho, una de las primeras medidas que se adoptó durante la etapa de la transición fue la del establecimiento del denominado como "ingreso irrestricto a las universidades nacionales". Este acontecimiento posibilitaría la apertura de la matrícula a todos los jóvenes en condiciones de continuar sus estudios en el nivel superior del sistema educativo argentino. En contraposición con la lentitud de ejecución de otros principios y medidas de política educativa, la del ingreso irrestricto se realizó inmediatamente en la práctica, aspecto que propició un notorio incremento de las tasas de matrícula universitaria en un lapso de tiempo muy breve. (BRASLAVSKY et al., 1989, p. 84).

Como muestra sucinta podrían valernos los siguientes datos: en 1950 la tasa bruta de educación universitaria para la cohorte 20-24 años era del 5.2\% y en 1988 del 38.4\%, lo

\footnotetext{
${ }^{5}$ En América Latina, la normalización se refiere al proceso mediante el cual las universidades públicas, después de un periodo de intervención de sus órganos de gobierno -consejos directivos, consejos superiores, asambleas universitarias- por parte de gobiernos dictatoriales, ponen en marcha los mecanismos de regularización de los padrones de los claustros - electores - y los mecanismos electorales para constituir dichos órganos de gobierno de acuerdo con lo establecido en sus estatutos. Vale destacar, además, que el gobierno de las universidades de la región se asienta en el principio del cogobierno de los diferentes claustros -profesores, estudiantes, graduados, personal no docente o de servicios- y se caracteriza por la alta participación de los diferentes sectores en la vida institucional y política de las instituciones de educación superior de la región. Un recorrido histórico y normativo acerca de la normalización de la Universidad argentina puede verse en Ruiz y Cardinaux (2010).

${ }^{6}$ La fecha 29 de julio de 1966 significó el inicio del proceso de intervención política de las universidades públicas por parte de los gobiernos civiles y militares que se sucedieron desde ese momento. La anulación de la autonomía universitaria fue la característica más notoria del período entonces iniciado y que se extendió hasta diciembre de 1983.
} 
cual significó el $100 \%$ el incremento de la matrícula entre los años 1980 y 1991. (SIGAL, 2003, p. 3). Los datos hablan por sí solos, en 1983 había 416.000 estudiantes universitarios, un año después llegaban casi a 500.000 y en 1986 superaban los 700.000. (BUCHBINDER; MARQUINA, 2008, p. 18). Dada la repercusión e importancia de esta cuestión, y el cambio tan sumamente drástico que supondría la irrupción de una educación superior de masas en la Argentina a partir de la instauración del acceso irrestricto, conviene, antes de adentrarnos en el análisis de prensa, conceptualizar de forma breve el concepto que es aquí objeto de estudio.

\section{El concepto de ingreso irrestricto a la universidad}

Durante la segunda mitad del siglo XX, las universidades argentinas experimentaron variaciones en sus modelos de acceso a sus estudios de grado ${ }^{7}$. En líneas generales, los cambios de políticas de ingreso irrestricto hacia políticas de acceso restrictivo fueron llevados adelante por gobiernos de facto en tanto que los cambios en el sentido inverso fueron promovidos por gobiernos democráticos de diferente orientación ideológica. (DUARTE, 2005, p. 2). En 1973 se implantó el ingreso irrestricto, es decir, sin ninguna prueba de admisión, sistema que se eliminó al comienzo del régimen militar en 1976, cuando se restableció el examen de ingreso y se impuso un cupo en las vacantes. Durante el periodo de transición a la democracia comenzó a repensarse y reclamarse por parte del movimiento estudiantil, y también por parte de algunos profesionales y especialistas, la reinstauración del sistema abierto de ingreso a las universidades, tal y como veremos en las siguientes páginas.

La denominada Comisión de Ingreso del Consejo de Rectores de las Universidades Nacionales - integrada por las autoridades de universidades públicas como las de Buenos Aires, La Plata, Centro, Rosario, Jujuy y Tucumán - trabajó durante el verano austral de 1982 para elaborar un dictamen que pudiera dar de paso al Ministerio de Educación. Sobre esa base se pretendía que se tomaran, en adelante, pautas generales, aunque "existía la intención de conceder mayores márgenes de iniciativa a cada casa de estudios". (LA NACIÓN, 22 de septiembre de 1982). La idea, señalaban sus defensores, se fundamentaba en que la situación era efectivamente muy diferente según cada universidad, dado que existían algunas en las que el número de vacantes era mayor al de aspirantes y otras en las que se daba el caso opuesto. Por su parte, por ejemplo, la Universidad Nacional de Rosario había recibido la autorización ministerial para llevar a cabo, con carácter de ensayo, un nuevo sistema de ingreso. Para ello, ofrecería a los estudiantes la posibilidad de recibir un curso en los meses de octubre, noviembre y diciembre, durante los sábados, a fin de no entorpecer las clases habituales de secundaria. Un segundo curso se desarrollaría en febrero y marzo y se incluirían "novedosos sistemas de evaluación y de probanzas vocacionales". (LA NACIÓN, 22 de septiembre de 1982).

Finalmente, en 1983, junto con el advenimiento de la democracia, se restableció el

\footnotetext{
${ }^{7}$ A diferencia de lo que ha acontecido en los estudios de grado, los estudios de posgrado en la Argentina (carreras de especialización, maestrías y doctorados) se caracterizan por tener mecanismos restrictivos: poseen cupos, son selectivos (a través de instancias de entrevistas, presentación de proyectos de investigación de tesis e incluso por medio exámenes de diferente tipo) y además en la amplia mayoría de los casos se trata de estudios arancelados. Todos los sistemas de ingreso a los estudios de posgrados son definidos por las universidades en particular, que suelen delegar su definición a las autoridades de los programas de cada posgrado en función de sus características académicas y sus propósitos formativos.
} 
ingreso irrestricto con modalidades de creciente diversificación, atendiendo a una fuerte demanda social reflejada por la movilización estudiantil. (FINOCCHIO, 2006). Entre 1973 y 1976, y a partir de 1983, predominan los casos de ingreso irrestricto sin preingreso ni concursos de apoyo y nivelación, aunque hubo varios casos en los que se vinculaban los cursos y exámenes no eliminatorios con el cursado de asignaturas del plan de estudios. En los casos en que existieron ciclos introductorios o de nivelación, estos duraban de uno a varios meses y tenían contenidos variables entre universidades y facultades. (SIGAL, 2003, p. 8-9). En consecuencia, una vez instaurada la democracia, la tendencia progresiva en el sistema de ingreso a las universidades en Argentina tendió hacia la implantación de criterios por parte de los establecimientos universitarios, sin que hubiera mayor regulación estatal salvo la posesión del título de nivel secundario. (RUIZ; SCHOO, 2014, p. 92).

Así, frente al estancamiento de la matrícula que se evidenció durante la época dictatorial, como resultado de la implementación de sistemas de cupos, aranceles o su desplazamiento al sector universitario privado o hacia el nivel terciario, el gobierno de Alfonsín otorgó prioridad al ingreso abierto a la universidad. Muchas universidades nacionales volvieron a establecer el ingreso irrestricto, aunque posteriormente comenzaron a implementarse los cursos de ingreso. Según Buchbinder y Marquina (2008, p. 27), "la expansión acelerada del número de estudiantes constituyó entonces, sin duda, una variable central de la historia universitaria del período de la transición democrática".

A pesar de las posibles diferencias que pudieran darse en el tipo de acceso a la universidad, como sostiene Braslavsky (1989, p. 83), el acceso irrestricto, traducido en términos prácticos, significó la posibilidad de que los jóvenes se inscribieran en la carrera que desearan, en cualquier universidad, para realizar allí sus estudios en forma gratuita y durante los años que quisieran. De hecho: En efecto, un elemento central de la transición democrática ha sido la recuperación
de la libertad para los distintos actores sociales. Pero como la posibilidad de
ejercicio de esa libertad está condicionada por los puntos de partida, los grupos
tradicionalmente más favorecidos de la sociedad han podido obtener más rápidas
ventajas del ejercicio de esa libertad. Es así como se ha resuelto mucho más
rápidamente el acceso irrestricto a las universidades nacionales que la erradicación
del analfabetismo y se han abierto caminos alternativos para contrapesar los efectos
de algunas medidas democratizadoras que permitieron a los sectores medios y altos
de la sociedad eludir eventuales pérdidas inmediatas, generando estrategias de
defensa de sus ventajas educativas comparativas. (BRASLAVSKY et al., 1989, p.
105).

Nos encontramos aquí con el debate o dicotomía tradicional en países desarrollados en cuanto a la prioridad del derecho a la educación o la libertad de enseñanza $^{8}$. Por un lado, políticas de ingreso libre se vinculan a procesos de democratización (traducidos en una mayor igualdad de oportunidades educativas) y, por el otro, políticas de ingreso denominadas como selectivas se asocian a la defensa de la calidad en la educación superior (traducidas en mecanismos meritocráticos para el ingreso

\footnotetext{
${ }^{8}$ El primero (derecho a aprender) refiere a una dimensión sustantiva del derecho a la educación como uno de los derechos fundamentales de los seres humanos, mientras que la segunda (libertad de enseñanza), si bien conforma uno de los contenidos del derecho a la educación, prioriza la atribución de las instituciones para definir sus contenidos curriculares. Asimismo, la libertad de enseñanza se vincula con el derecho al trabajo de los docentes, es decir, con la libertad de cátedra de los profesores para enseñar de acuerdo con sus orientaciones científicas, pedagógicas e ideológicas.
} 
a los estudios superiores, la permanencia en ellos e incluso el otorgamiento de becas de estudios). En este contexto, la universidad de la transición se preocuparía rápidamente por la introducción de políticas de acceso democratizantes (irrestricto y gratuito), aunque pronto debería ocuparse también por resolver las consecuencias de un ingreso masivo de estudiantes en condiciones académicas de gran heterogeneidad. En el momento de la transición, la lucha estudiantil por la consecución del acceso irrestricto a las universidades significaría un eslabón más en la consecución de la libertad, pero el cambio generado por esa política de acceso obligaría a enfrentar distintos desafíos y dificultades a los rectores de turno. Así, por ejemplo, relata Buchbinder (2005, p. 215):

El rector normalizador de la Universidad de Buenos Aires, Francisco Delich, al señalar, poco tiempo después de asumir su gestión, los principales problemas que debía afrontar, manifestaba que se encontraba con una Universidad de masas, prácticamente sin investigación, con sus orientaciones profesionalistas profundamente acentuadas, inmersa en un proceso de deterioro de la formación de sus docentes y con graves problemas edificios, agravados por la explosión que había experimentado la matrícula durante ese mismo año 1984.

Desde 1983 hasta 2017, el ingreso irrestricto a la universidad en Argentina ha generado debates, polémicas diversas y confrontaciones de política educativa. Es más, como ejemplo más reciente puede mencionarse un caso del año 2016 al que refiere una nota aparecida en Clarín digital, bajo el siguiente titular: "La Justicia bloqueó el ingreso irrestricto a la universidad". (CLARíN, 12 de mayo de 2016). Mediante sentencia judicial, la disposición sobre ingreso irrestricto contemplada en la enmienda a la Ley de Educación Superior № 24.521 (dispuesta por la ley № 27.204 de 2015) quedaba anulada. El argumento del juez sostenía que dicha enmienda legislativa violaba la autonomía de las instituciones universitarias en lo que concierne al establecimiento de sus sistemas de ingreso y régimen de estudios ${ }^{9}$. No entraremos en el análisis de este artículo periodístico sobre las consideraciones actuales del asunto, dado que nuestra intención es relatar (mediante el sondeo de la prensa de la época de la transición) la actitud y las acciones del movimiento estudiantil en su lucha por la consecución del acceso irrestricto a las universidades. Sin embargo, mencionamos este artículo de 2016 como ejemplo de la plena actualidad que mantiene el tema en el debate político educativo de Argentina.

\section{El acceso universitario irrestricto en los discursos de la prensa diaria}

El desenlace de la Guerra de Las Malvinas propició la aprobación de una de las primeras medidas tendentes a la apertura del ingreso en las casas de estudio a los soldados combatientes. La Prensa anunciaba los días 25 y 26 de junio de 1982 la decisión del Ministerio de Cultura y Educación de facilitar, en todo lo posible, el trámite de ingreso a los alumnos afectados por la convocatoria militar, sin que ello implicase alterar las disposiciones fundamentales. Se trataba de "otorgarles facilidades para normalizar su vida

\footnotetext{
${ }^{9}$ A partir del año 1994, tras la reforma de la Constitución Argentina, la autonomía universitaria se convierte en una garantía constitucional ya que la carta magna de Argentina la establece y dispone que toda la legislación educativa debe respetar la autonomía y autarquía de las universidades nacionales. Por lo tanto, no corresponde al Poder Legislativo Nacional la fijación de los límites de la autonomía universitaria dado que ella está garantizada por la propia Constitución Nacional. Una fuerte controversia se desató a partir lo dispuesto por la ley aprobada en 1995 (ley № 24.521 de Educación Superior, aún vigente) dado que parte de su articulado cercena la autonomía consagrada en la Constitución Nacional, reformada en el año previo.
} 
estudiantil", se decía en Clarín el 25 de junio de 1982. La Nación, por su parte, iba más allá en la valoración de esta primera medida considerada como, "apenas, el principio de un fenómeno social cuya dimensión exacta sólo a partir de ahora comenzaría a advertirse". (LA NACIÓN, 30 de junio de 1982). Para Bartolomé Mitre, redactor del artículo, esos estudiantes, excombatientes en las Malvinas, pronto deberían enfrentarse a la votación en sus primeros actos comiciales, siendo ahora ciudadanos de derechos y no sólo de deberes, como hasta el momento les había tocado vivir por la responsabilidad asumida en la guerra. Ello les convertía en un "caudal electo virgen" que los partidos políticos y sus dirigentes querrían aprovechar. (LA NACIÓN, 30 de junio de 1982). Diferentes discursos sobre lo que en realidad era un mínimo paso de apertura al ingreso, adoptado sólo como medida provisoria como consecuencia de lo que representa una cruenta guerra, pero que daría, metafóricamente, el "pistoletazo de salida" a lo que serían las demandas por la introducción de un sistema de ingreso abierto a las universidades argentinas que se producirían durante meses sucesivos.

Desde el primer momento, las peticiones de aprobación de un sistema de acceso irrestricto a las universidades estuvieron estrechamente ligadas a la movilización estudiantil de la Argentina del periodo de la transición a la democracia. El petitorio del estudiantado, reflejado en los tres periódicos manejados, referiría cuestiones tales como la recuperación del estado de derecho, el esclarecimiento de la situación de los "compañeros desaparecidos", la libertad de los detenidos sin causa ni proceso, la derogación de la ley de facto para las universidades aprobada en 1981 "por antidemocrática y antipopular", el inicio de una política social y cultural de liberación nacional, el acceso abierto e irrestricto a la universidad, la eliminación de todo tipo de arancelamiento... (LA PRENSA, 16 de marzo de 1983). De hecho, varios de estos aspectos coinciden con lo que para William Leslie Chapman constituían las expresiones públicas que se leían en la mayor parte de periódicos, formuladas por políticos y profesionales de la educación, respecto a lo más aconsejable para el gobierno y el quehacer de las universidades argentinas y que se vinculaban con cuatro medidas fundamentales: a) Ingreso irrestricto (para el propio Chapman inasumible en aquel momento, tal y como manifestará en otras ocasiones a las que nos referiremos más adelante); b) Eliminación de aranceles; c) Anulación de los concursos docentes realizados durante los años de la dictadura militar; d) Gobierno tripartito (de los claustros) en las universidades. (LA NACIÓN, 27 de junio de 1983).

Sin embargo, el discurso reflejado en los periódicos La Prensa, Clarín y La Nación respecto a los reclamos realizados mediante movilizaciones y actuaciones estudiantiles de diversa índole y, especialmente, a las formas y modos en que éstos se hacían, diferirá notablemente entre ellos. Para La Prensa, diario de talante conservador, las actuaciones estudiantiles no discurrían por las vías oportunas y se asociaban a reclamos de entidades peronistas y comunistas, ante las que se mostraba en clara oposición. De hecho, ante el propósito de reconstitución de la FUA durante el verano de 1982, el diario ya se había mostrado totalmente en contra al considerar que la iniciativa, "en la que tendrán activa intervención los comunistas y los socialistas populares, será un refugio ideal para los extremismos de todo cuño y el populismo vernáculo" y los "lemas demagógicos como el de "llevar la universidad al pueblo", alcanzarán fácil difusión en el abigarrado conglomerado estudiantil, y no faltarán quienes exijan la reforma de los programas o la renuncia de aquellos profesores que les resulten desafectos". (LA PRENSA, 31 de agosto de 1982). 
Máximo Gainza, redactor de todos los documentos de este diario que han sido manejados en este estudio, acusaba a los grupos de movilización estudiantil de acciones subversivas y terroristas que estaban convirtiendo a las universidades en "centros de agitación permanente y en escenarios de enconadas luchas banderizas". (LA PRENSA, 16 de marzo de 1983). Asimismo, calificaba algunas de estas protestas como improcedentes y defendía el sistema de acceso y de cupos vacantes como mecanismo adecuado de selección para el ingreso a las universidades. Así lo hacía ante una protesta acontecida en Rosario durante el mes de septiembre de 1983 y que llevó a 30 estudiantes secundarios a declararse durante días en ayuno, en plena calle y frente al Rectorado, hasta que obtuvieran medidas especiales para el ingreso a la universidad. Los alumnos habían aprobado sus exámenes de ingreso, pero sus calificaciones no habían sido lo suficientemente altas como para entrar en los cupos fijados para cada facultad. A su protesta se unieron los padres y otras personas que, al pasar por la calle y tener noticia de la situación, decidieron comenzar a recabar firmas para suscribir un petitorio que solicitara la renuncia del rector Humberto Riccomi. La protesta adquirió tal magnitud mediática que motivó declaraciones de diversas entidades políticas en las que se formulaban críticas al sistema universitario vigente y se aboga por el ingreso irrestricto a la Universidad. Sin embargo, para el diario en cuestión, la protesta estudiantil resultaba improcedente y se justificaba la existencia del sistema de cupos vacantes como un procedimiento nada arbitrario ni elitista sino como un mecanismo que obedecía a razones prácticas vinculadas a las posibilidades de las casas de estudio. (LA PRENSA, 24 de septiembre de 1983).

En el caso de Clarín, el discurso resulta mucho más moderado que el anterior e, incluso, demuestra un posicionamiento favorable respecto a las peticiones estudiantiles, llegando a argumentarse por parte de Jorge Abásolo, redactor de la mayor parte de los documentos manejados, que los reclamos estudiantiles no "parecerían estar tan lejos de las aspiraciones de todos los partidos políticos, por más que existan controversias inevitables entre ellos". (CLARÍN, 27 de octubre de 1982). Se reconoce además que, a partir del conflicto de las Malvinas, "todo el país expresó cada vez más nítidamente una inclinación política que, en el terreno universitario, se manifiesta en la agremiación y politización de los estudiantes" (CLARíN, 8 de diciembre de 1982), aspecto que nos haría inferir que se entiende lógico el clima de efervescencia estudiantil. De este modo, Clarín se va a convertir en el principal diario que vehicula y transmite a la opinión pública informaciones sobre ese movimiento estudiantil que reclamaba una serie de cambios imprescindibles para el buen funcionamiento universitario. Un movimiento que estaría, a su vez, estrechamente ligado con la reorganización de grupos, federaciones y agrupaciones de alumnos, de tipo diverso, que iba a adquirir especial fuerza durante el periodo de referencia. De hecho, junto al acceso irrestricto, el asunto de las elecciones estudiantiles cobró notable importancia en la prensa de la época analizada, especialmente en el diario referido. Para Ernestina Herrera de Noble, directora de Clarín, esta cuestión resultaba del todo positiva y era la representación de otras muchas cosas que se vivían en el país:

Después de siete años de una virtual congelación de lo que podría llamarse la actividad política interna de los estudiantes universitarios, es reconfortante comprobar cómo la lucha y el debate de las diferentes tendencias ha resurgido en un ambiente de convivencia y pacífico disenso, sin que ninguna manifestación de violencia ni gesto desaforado alguno hayan venido a perturbar esta lenta normalización. [...]. La pluralidad, el ámbito de convivencia y la voluntad de 
participación que han erigido los comicios en las universidades constituyen un excelente ejemplo para la futura etapa que ha de vivir el país, tan necesitado de pasión, lucidez y tolerancia. (CLARín, 14 de junio de 1983).

Sin embargo, no podemos confundir el clima más o menos tranquilo en el que se habrían desarrollado esas elecciones estudiantiles con la ausencia de concentraciones y manifestaciones estudiantiles durante todo este periodo, y repartidas por diferentes lugares del territorio argentino, que además habrían sido coincidentes en la mayor parte de sus reclamos. Para Abásolo, inicialmente las diferencias en relación con el movimiento de épocas anteriores (en clara referencia al periodo de 1966-1976), tanto por el estilo como por el contenido de los textos que circulaban por manos de los alumnos, eran notables. El discurso que transmitía este diario a la opinión pública en relación al movimiento estudiantil se enfocaba a minimizar los efectos de la actuación de los alumnos, alegando que se había pasado de la promoción de la insurrección a "reuniones de alumnos cuyos planteos políticos no manifiestan tan alto encono". (CLARÍN, 17 de noviembre de 1982). Tal como sostiene Arriondo (2011), la década de 1980 se caracterizó por una presencia renovada del movimiento estudiantil, en referencia al decrecimiento de la radicalización política que se inició a finales de la década de 1960. Sin embargo, esto no quiere decir que durante el marco cronológico estudiado no hubiera varias situaciones de mayor conflictividad, aunque la lucha ya no fuese tan solo política sino también educativa, enfocada además en aspectos tales como el acceso a la universidad.

De nuevo el periódico Clarín, el 6 de octubre de 1982 y bajo el titular "Vuelve el movimiento estudiantil", anunciaba que los estudiantes se mostraban cada vez más animados a desarrollar sus propias organizaciones representativas. En este sentido, se aportaban datos sobre la situación que se vivía en las diferentes facultades de la Universidad de Buenos Aires (UBA). El clima de agitación y de demandas democratizadoras, aunque siempre con un carácter pacífico, aumentó y dio lugar a distintas manifestaciones, declaraciones y documentos con el reclamo de la supresión del arancelamiento de los estudios, de los cupos al ingreso y otras cuestiones similares. Para marzo de 1983, se habían efectuado ya varios estudios y análisis sobre el tema de los cupos de ingreso, lo que habría llevado al Estado a propiciar un aumento general del 10\% en el número de plazas ofrecidas en todo el país; sin embargo, facultades como Medicina o Arquitectura conservarían las mismas plazas para ese curso académico. En el periódico Clarín (16 de marzo de 1983) podemos leer que esta medida no dejaría satisfecho al movimiento estudiantil, pues su petición era la de la anulación de los cupos. Una vez más, el diario se mostraba cercano a las peticiones efectuadas por los grupos estudiantiles.

En el caso de La Nación, estamos ante el diario más mesurado en cuanto a la cuestión de la movilización estudiantil y la reorganización de grupos, federaciones y agrupaciones de alumnos. Con frecuencia, el discurso se orienta a informar de acontecimientos en este sentido, a restar importancia o minimizar el impacto del clima de agitación estudiantil ante la opinión pública y, en ocasiones, a alertar a la sociedad sobre el posible radicalismo estudiantil, representado para su director por Franja Morada, una agrupación estudiantil que, reuniendo a anarquistas, socialistas y radicales, habría intentado nuclear a las fracciones reformistas no comunistas. (BELTRÁN, 2013, p. 38). La opinión de Bartolomé Mitre sobre el clima estudiantil queda claramente reflejada en un editorial en el que considera que lo que define a la universidad como institución, y a los 
miembros que la integran, no es otra cosa que la voluntad de búsqueda de la verdad a través de la razón. Considera que, después, se podrán tener todas las discrepancias ideológicas, políticas o académico-científicas que se quiera, pero el punto de partida común será siempre esa actividad racional que, desde su particular punto de vista, se estaría viendo quebrantada en numerosas ocasiones por las actitudes y manifestaciones de algunas agrupaciones de estudiantes. (LA NACIÓN, 20 de abril de 1983).

A pesar de ser La Nación el diario donde el discurso sobre el acceso irrestricto a las universidades se muestra más comedido o, incluso, opuesto a él en los primeros meses de la transición, con el devenir del tiempo se reconducirá hacia la exposición de cierta pluralidad de posiciones e, incluso, llegará a mostrar posturas a favor del mismo. A través de los discursos emitidos en este diario es posible reconstruir la crónica de sucesos de la época en relación a la cuestión que nos ocupa, constituyendo la principal fuente de referencia, siendo las alusiones en los otros dos diarios proporcionalmente menos frecuentes. Así, por ejemplo, en el mes de febrero de 1983, La Nación se hacía eco de una declaración de Franja Morada, agrupación estudiantil que iba progresivamente ganando mayor peso entre buena parte de estudiantes, de la cual se decía que sería interpretada desde las esferas académicas como un toque de atención en relación con el pulso que podía tomar el movimiento estudiantil en adelante. Mitre consideraba que habría roto "el fuego del movimiento estudiantil este año, pero sin que su contenido refleje otros puntos de vista que los ya conocidos de esta agrupación [...]: la anulación de los concursos docentes (ejecutados durante la dictadura militar), el ingreso irrestricto en las casas de estudios y las referencias al tema de los desaparecidos como puntos sobresalientes". (LA NACIÓN, 16 de febrero de 1983). Además de estas cuestiones, el documento contenía una propuesta aunque no abundaba en detalles - para solucionar la desarticulación entre el nivel secundario y el nivel superior universitario y llamaba a la unidad y movilización de los sectores allegados al ámbito universitario animados por el lema "Reconquistemos la Universidad para el pueblo argentino".

Casi de forma coincidente a la anterior declaración alzó su voz la Unión Nacional de Estudiantes para, según La Nación (16 de febrero de 1983), "rechazar la opción facilista y demagógica del acceso irrestricto tal y como había sido concebido hasta el momento". Esta agrupación reconocía el ingreso irrestricto como una opción válida, pero siempre y cuando se cumplieran algunas pautas fundamentales, entre ellas mejorar la educación primaria y secundaria mediante una reestructuración del sistema.

Manifestaciones y reclamaciones de los estudiantes por el acceso irrestricto se harían patentes en diferentes momentos durante los últimos meses de 1982 y, especialmente, al inicio del curso 1983. Con el paso del tiempo, la sensación de estar siendo desoídos por parte de las autoridades crecía entre el estudiantado y con ello aumentaban sus ganas de manifestarse. Veamos simplemente un dato que resulta bastante clarificador de la situación que la limitación del acceso estaba provocando en aquellos jóvenes, tal y como refería el diario en cuestión. Al comienzo del curso 1983, de un total de 1500 alumnos que se presentaron al examen de ingreso para la carrera de Psicología de la UBA aprobaron 1330, de los cuales sólo ingresaron 268 que era el cupo prefijado. Muchos de esos estudiantes se concentraron en el patio del edificio de la institución, lo que constituyó la primera muestra de disconformidad por la limitación de vacantes. El siguiente paso consistió en la interposición de un recurso de amparo ante la 
Justicia para que se realizara una revisión pública de los exámenes, a lo que el entonces Ministro de Educación, Cayetano Licciardo, alegó que lo que se había hecho en Psicología no era otra cosa que "la selección de los talentos" (LA NACIÓN, 13 de abril de 1983), dando muestra clara de un sistema meritocrático y no equitativo. Sin duda, la opinión de las autoridades del momento servía como argumentación del discurso proferido en La Nación.

Aun siendo noticiables para este diario este tipo de reclamaciones estudiantiles, la tendencia, como ya se ha mencionado anteriormente, era la de minimizar y transmitir al público lector una sensación de tranquilidad y calma en el clima universitario. Según Bartolomé Mitre, el curso académico correspondiente al año 1983 se había iniciado con un clima distinto. En la UBA parecían ser inaudibles los ecos del zarandeado tema de los exámenes de ingreso y la limitación de vacantes, aunque decía aquél que "seguramente seguirán dando pábulo a una polémica que llegará probablemente a su fin cuando se adopte una solución de fondo". (LA NACIÓN, 6 de abril de 1983). No se equivocaba, pronto las protestas estudiantiles darían perfecta muestra de ello.

A principios de marzo, ante el inminente inicio del curso escolar, la Federación Universitaria de Buenos Aires (Fuba) se concentró a las puertas del Ministerio de Educación para reivindicar la supresión del examen de ingreso, la derogación de cupos y el aumento del presupuesto universitario ${ }^{10}$. Las estimaciones del recuento de manifestantes oscilaron de sólo los 200 a los 2000 y no se obtuvo una respuesta inmediata a las peticiones, pero esta concentración, de carácter pacífico y ordenado, "marcó la temperatura política para el nuevo curso en el ámbito universitario". (LA NACIÓN, 16 de marzo de 1983).

De hecho, la Fuba tuvo un papel fundamental en la reivindicación del acceso irrestricto a la universidad. Durante los años de la transición democrática, la federación buscaba su reubicación política en el contexto institucional y se mostró del todo partidaria por la consecución de este fin, no sin oposición y obstáculos constantes a sus reivindicaciones político-estudiantiles. A finales del año 1983, y tras la celebración del congreso de la Fuba, su entonces presidente, Andrés Delich (hijo del rector normalizador de la UBA durante esa misma época), declararía lo siguiente: "Si bien es política de la Federación la constante lucha por el acceso irrestricto a la universidad, pediremos la mayor flexibilidad en el sistema y, en todo caso, el examen será la excepción y no la regla". (LA NACIÓN, 16 de noviembre de 1983).

Pero no sólo la Fuba se adheriría a este tipo de reclamos, pues la mayor parte de organizaciones estudiantiles que comenzarán a reagruparse o fundarse tras el fin de la Guerra de Las Malvinas, a excepción de grupos mínimos, coincidirán "con exigencias de coyuntura: cese de los concursos, quita del arancel, ingreso irrestricto, falta de nivel académico y eliminación de las fuerzas de seguridad en las facultades". (LA NACIÓN, 19 de enero de 1983).

En una reunión celebrada por el Consejo de Rectores de Universidades Nacionales (Crun) durante el mes de abril de 1983 se deliberaron, a puerta cerrada, varios asuntos universitarios, entre ellos el del acceso a las universidades. Así, el vicepresidente del CRNU, el doctor Riccomi, anunció que, durante el siguiente curso, en todas las casas de

\footnotetext{
${ }^{10}$ La Fuba es la confederación que agrupa a todos los centros de estudiantes de las diferentes unidades académicas (Facultades) que componen la Universidad de Buenos Aires. Junto con las otras federaciones de estudiantes universitarios, de las demás universidades públicas, conforma la Federación Universitaria Argentina (FUA).
} 
estudio se aplicaría un curso de ingreso con características todavía por determinar. En el artículo de prensa de referencia se decía que este tipo de procedimiento alejaba el riesgo de la selección con examen único y brindaba mayores posibilidades de evaluación acordes con las aptitudes del aspirante. En rigor, se argumentaba, este esfuerzo buscaba solucionar el problema del ingreso,

[...] cuya responsabilidad mayor les cabe a las autoridades que desde 1976 no encontraron la forma de llevar a cabo sus intenciones a buen término. [...] En aquel entonces se apeló al examen de ingreso como solución de emergencia para reemplazar el sistema de acceso irrestricto que regía desde 1974. (LA NACIÓN, 27 de abril de 1983).

Los problemas en torno a la cuestión del acceso a la universidad siguieron sucediéndose. Durante el mes de mayo de 1983 se dio a conocer a través de La Nación (18 de mayo de 1983) que la UBA iba a autorizar a la Facultad de Arquitectura y Urbanismo a fijar un puntaje inferior al establecido originariamente para el ingreso, de manera que pudiera admitir a una cantidad mayor de aspirantes (unos 170 en este caso concreto) que habían aprobado el examen, pero carecían de vacantes. Las reclamaciones de los estudiantes en esta ocasión fueron reforzadas por una buena parte de profesores de la facultad, reunidos en audiencia con el rector Carlos Segovia Fernández. Para el autor del escrito, la situación resultaba discriminatoria para otras facultades en las que también por coincidencia de notas se habían concedido más vacantes que no podrían ser finalmente cubiertas, como eran Ciencias Veterinarias, Farmacia y Bioquímica, Medicina, Odontología o la carrera de Psicología (esta última estaba bajo la órbita del Rectorado ${ }^{11}$ ). El asunto revestía verdadera complejidad.

Un mes después, un grupo de padres comenzó a desarrollar una intensa acción con miras a que sus hijos, aspirantes a estudiar en la UBA - con examen aprobado, pero sin plazas vacantes - pudieran ingresar en ese momento. La movilización se desarrolló a través de comisiones por facultades, de forma similar a la que había sucedido previamente en Rosario. Los padres sostenían en todo momento que carecían de objetivos políticos y que lo único que les movía era el respeto por el derecho de aprender de sus hijos. Pero los problemas para dar solución a esta situación eran varios: para el curso 1983, ya bastante avanzado en ese momento, se habían aumentado de forma notoria el número de plazas ofertadas. De hecho, para el entonces rector, Humberto Riccomi, estaban "al límite máximo que cada facultad puede absorber aun -puntualizó- en detrimento del esfuerzo y sacrificio que realizan los docentes frente a una población estudiantil que excede en mucho las posibilidades de aulas, laboratorios y recursos humanos disponibles". (LA NACIÓN, 15 de junio de 1983). A pesar de todo, de acuerdo con la información de los círculos académicos, las autoridades de la UBA estaban dispuestas a ceder parcialmente al requerimiento de los padres, al menos en aquellas facultades con cursos especiales, de acuerdo a lo que ya se

\footnotetext{
11 La carrera de Psicología de la UBA fue creada en el año 1957, junto con otras como Sociología, Ciencias de la Educación y Ciencias Antropológicas, en un período de profunda renovación académica de la UBA. Todas ellas estaban en la Facultad de Filosofía y Letras. Como una de las consecuencias de las políticas represivas iniciadas en 1976, las carreras de Sociología y Psicología fueron extraídas de su Facultad de origen y puestas bajo la órbita directa del rector interventor de la UBA, como forma de ejercer un control estricto y autoritario. Por ello, para el inicio del proceso de transición democrática estas dos carreras universitarias no se encontraban dentro de ninguna unidad académica. En el año 1985 se crearía la Facultad de Psicología y en el año 1988 la de Ciencias Sociales, que incluye a la carrera de Sociología.
} 
había hecho, como mencionamos anteriormente, en otras facultades. Es decir, en esta ocasión la flexibilización de los cupos podría aplicarse en dos unidades académicas, la de Derecho y Ciencias Sociales y la de Ciencias Exactas y Naturales.

De hecho, el artículo de La Nación que referenciábamos antes nos aporta una de las claves básicas de la situación en la que se encontraba, a mediados del año 1983, el proceso de inserción de ingreso irrestricto. Decía lo siguiente:

Según fuentes universitarias, admitir a todos los aprobados en esta ocasión no será más que anticipar el temperamento que se aplicará el año próximo en los cursos especiales - por ampliarse a otras facultades y universidades - que apunta a alejar el riesgo de la selección mediante examen único y brindar mayores posibilidades de evaluación, acordes con las aptitudes de cada aspirante. (LA NACIÓN, 15 de junio de 1983).

El sistema de examen de ingreso y cupos en las vacantes instaurado en 1976 establecía unos mecanismos de acceso muy alejados de la igualdad de oportunidades educativas que debería caracterizar a todo entramado democrático. Incluso la prensa se hacía eco de ello con motivo de la celebración de los exámenes de ingreso para el curso 1983, considerando que "los cupos no siempre tuvieron que ver con la vocación y capacidad de quienes aspiraban a entrar en la universidad. Sin lugar a dudas que lo más justo sería que aquel que demuestre capacidad para afrontar con posibilidades de éxito los estudios superiores, cuente con un lugar en las aulas de las facultades". (LA NACIÓN, 23 de marzo de 1983). Como vemos, la posición del diario La Nación parecía comenzar a flexibilizarse. De nuevo en mayo de 1983 tenemos noticia de una reclamación, efectuada por el presidente de un centro estudiantil universitario (sin determinar), relacionada con errores en el curso de ingreso. En esta ocasión se denunciaba la inclusión de asignaturas que nada tendrían que ver con el plan de estudios y que, según él, se habrían incluido "con un fin tendencioso, para que fueran un filtro y obligaran a la deserción". (LA NACIÓN, 20 de mayo de 1983).

A pesar de que a mediados del año 1983 los reclamos estudiantiles, y las opiniones manifestadas tanto en prensa como en otros foros de especialistas, apuntaban al necesario e inminente establecimiento del acceso irrestricto a las universidades, todavía podemos leer opiniones contrarias al mismo en La Nación. Este es el caso, por ejemplo, de un artículo de opinión de William Leslie Chapman, Decano de la Facultad de Ciencias Económicas de la UBA (1958-62) y Vicerrector de esta universidad (entre 1960-62). En relación con el ingreso irrestricto -pues también hablaba de la eliminación de aranceles, la anulación de los concursos y el gobierno tripartito en las universidades-, Chapman consideraba que ya era "hora de que desmitifiquemos la necesidad de estudiar en la Universidad, comúnmente concebida como un desiderátum inigualable en países de pobre desarrollo cultural". (LA NACIÓN, 27 de junio de 1983). Asimismo, atacaba a esa creencia generalizada que consideraba que la selección de los más aptos para el estudio debía hacerse una vez producido el ingreso y no antes. Para Chapman, la experiencia había demostrado que, de este modo, se derrochaban recursos escasos y se perdía "tiempo en intentar la formación de quienes carecen de condiciones para aquel nivel de estudios". (LA NACIÓN, 27 de junio de 1983). En consecuencia, si se aprobaba el funcionamiento de este nuevo sistema de acceso, el problema que tendrían que atender quienes gobernasen las universidades en 1984 sería el de "cómo atender eficientemente a una enorme masa de estudiantes, cuando 
desde hace mucho tiempo faltan recursos humanos para hacer frente a la gran demanda que se arrastra de año en año". (LA NACIÓN, 27 de junio de 1983).

El tiempo transcurría y el mes de septiembre de 1983 evidenciaría el pico más alto de protestas estudiantiles, con marchas, concentraciones, huelgas de hambre, cierre de facultades y renuncia de funcionarios que, según La Nación (21 de septiembre de 1983), representaban "signos inquietantes de estos momentos". De este modo se llegaba a los últimos meses del año 1983, todavía con la tarea pendiente por parte de las autoridades de definir la política de ingreso para el año próximo. (LA NACIÓN, 2 de noviembre de 1983). Previamente, había tenido lugar la celebración de un Seminario sobre transferencia del ciclo secundario a la universidad, organizado por el Consejo de Rectores de Universidades Nacionales y la Organización Universitaria Interamericana, y en él se habían presentado estudios, opiniones e informes diversos sobre la cuestión. La ampliación de la capacidad de admisión del sistema universitario seguía presentando, de acuerdo al discurso recogido en La Nación (17 de agosto de 1983), grandes controversias.

Finalmente, el comienzo del curso 1984 coincidió con la aprobación de un sistema de acceso abierto a las universidades argentinas, por lo que cada una de ellas estableció la modalidad de ese acceso, normalmente gestionados mediante cursos especiales o ciclos básicos comunes que permitirían la posterior continuación de los estudios en las Facultades. La fuerza que los reclamos estudiantiles jugaron durante la etapa de la transición resulta evidente a partir de los datos expuestos, pero el principal problema es que este cambio no fue acompañado de otros, tales como: la adecuación curricular; la aplicación de sistemas de tutorías para los estudiantes provenientes de los sectores más empobrecidos, de modo que se garantizara no sólo su ingreso sino también su permanencia y graduación; la planificación de la oferta de carreras de grado y la promoción de la cooperación inter-institucional para evitar la masificación excesiva de los cursos; el aumento presupuestario para el sector universitario, etc. En tal sentido, algunos autores sostienen que este cambio de la política de ingreso aconteció sin la generación de condiciones mínimas para contener la fuerte expansión de la matrícula universitaria que se evidenció en la última mitad de la década de 1980. (CHIROLEU, 2009, p. 152). Sin embargo, es necesario destacar que muchos de esos problemas (como la ausencia de la planificación del crecimiento y la escasez de recursos para la universidad) se debieron a razones estructurales de crisis de la economía y de la política argentina luego del período dictatorial que dejó profundas secuelas en la organización del Estado argentino, así como una extraordinaria deuda externa, además de problemas de gobernabilidad debido al proyecto represivo autoritario que debilitó las instituciones políticas y sociales del país. Todo ello, conjuntamente con el dificultoso contexto político que afrontó el gobierno constitucional que asumió en diciembre de 1983, contribuyó a que algunas de sus iniciativas más auspiciosas, como el ingreso irrestricto a la universidad (que fue acompañado por una medida idéntica para el nivel secundario del sistema educativo), no pudieran ser del todo efectivas en términos de garantizar el goce del derecho a la educación para el conjunto de la población.

\section{Conclusiones}

El movimiento estudiantil argentino de la época de la transición a la democracia (1982/83) desarrolló un rol fundamental en la consecución del acceso abierto a las 
universidades. Tal como hemos visto, fueron constantes y crecientes las reclamaciones y manifestaciones pacíficas del estudiantado por un sistema de acceso irrestricto a la universidad durante la época. Sin embargo, los discursos vertidos por la prensa diaria no coincidieron en sus posiciones respecto al asunto - habiendo podido influir, en consecuencia, de modo diferenciado en la opinión pública -, pudiéndose apreciar claros matices diferenciadores entre los tres diarios analizados. Para La Prensa, no sólo las protestas estudiantiles resultaban improcedentes, sino que el sistema de acceso restringido y de cupos vacantes constituía un mecanismo adecuado de selección para el ingreso a las universidades. El caso de Clarín es contrario, mostrándose favorable respecto a las peticiones estudiantiles. Por su parte, el diario La Nación plasmará un discurso sobre el acceso irrestricto a las universidades mucho más conservador o comedido en los primeros meses de transición a la democracia que, sin embargo, dará paso, con el tiempo, hacia la exposición de cierta pluralidad de posiciones e, incluso, llegará a mostrar posturas a favor del mismo. De cualquier forma, aunque desde diferentes posturas, todos ellos se hacen eco de la cuestión estudiada, entendiendo que reviste cierto interés social para la opinión pública.

Lo cierto es que, tal y como apuntan algunos autores, con el transcurso de los años, esa demanda de los estudiantes acabaría por constituirse -en el imaginario social-en una de las características del sistema de educación superior argentino como "democrático" en términos de igualdad de acceso de cualquier ciudadano que quisiera seguir estudios de nivel superior. (GESSAGHI; LLINÁS, 2005, p. 13). De esta forma, aquella lucha estudiantil significaría un eslabón más en la promoción del derecho a la educación a través del acceso a una educación universitaria en condiciones de mayor equidad.

Ahora bien, aunque no es el objeto de estudio de este artículo, no podemos dejar de señalar que el cambio generado por esa política de ingreso irrestricto a la educación universitaria obligaría a enfrentar en el futuro distintos desafíos y dificultades que acarrearían problemas para el conjunto del sistema. El problema principal vino dado porque a esas demandas estudiantiles por un ingreso irrestricto no le seguiría el necesario aumento de la inversión en recursos humanos y materiales. Tampoco se evidenciaron cambios en el conjunto del sistema que tornaran factibles la continuación de los estudios universitarios para los estudiantes de los sectores más empobrecidos que comenzaron a ingresar a la universidad.

Con todo, se puede evidenciar el alto nivel de implicación que tuvo el movimiento estudiantil argentino en la consecución del acceso irrestricto a las universidades nacionales durante la etapa de la transición, así como el traslado de todo ese proceso a la opinión pública a través de la prensa de la época. Su rol como promotor de cambios democráticos en una sociedad que había estado sometida a un proyecto político represivo fue sustantivo y en tal sentido promovió demandas cualitativamente distintivas que dejaron su impronta en el sistema educativo en su conjunto. Muchas de aquellas demandas - por una universidad autónoma más equitativa e igualitaria - fueron resignificadas a la luz de la reconversión político-educativa neoliberal que se instauró en la década siguiente.

\section{Referencias}

ARENDT, Hannah. La condición humana. Barcelona: Paidós, 1993. 
Sobre la revolución. Madrid: Alianza editorial, 1998.

ARRIONDO, Luciana. Universidad y Política: el movimiento estudiantil en los 80. La revista del CCC [en línea], n. 11, enero/abril 2011. Disponible en:

<http://www.centrocultural.coop/revista/articulo/209>. Acesso en: 6 nov. 2016.

BELTRÁN, Mónica. La Franja. De la experiencia universitaria al desafío del poder.

Buenos Aires: Aguilar, 2013.

BRASLAVSKY, Cecilia et al. Educación en la transición a la democracia. Casos de Argentina, Brasil y Uruguay. Santiago, Chile: UNESCO-OREALC, 1989.

BUCHBINDER, Pablo. Historia de las universidades argentinas. Buenos Aires: Editorial Sudamericana, 2005.

BUCHBINDER, Pablo; MARQUINA, Mónica. Masividad, heterogeneidad y

fragmentación: El sistema universitario argentino 1983-2008. Buenos Aires: Universidad Nacional de General, Sarmiento, 2008.

CANTINI, José Luis. La autonomía y la autarquía de las universidades nacionales. Buenos Aires: Academia Nacional de Educación, 1997.

CARRILLO-LINARES, Alberto. Universidades y transiciones políticas: el caso español en los años 60-70. Espacio, Tiempo y Educación, v. 2, n. 2, p. 49-75, 2015.

DUARTE, Betina. El acceso a la Educación Superior: Sistemas de Admisión a las Universidades Nacionales de Argentina. Primer congreso de la Sociedad Argentina de Estudios Comparados en Educación, 2005. Disponible en:

<http://www.saece.org.ar/papers1.php>. Acceso en: 28 sept. 2016.

FINOCCHIO, Silvia. La Historia de la educación en los debates políticos actuales en América latina: un estudio desde la prensa. Espacio, Tiempo y Educación, v. 3, n. 1, p. 317-340, 2016.

GESSAGHI, Victoria; LLINÁS, Paola. Democratizar el acceso a la educación superior. Documentos de trabajo del Centro de Implementación de Políticas Públicas Para el Equidad y el Crecimiento, 2005. Disponible en: <http://www.cippec.org/-/democratizarel-acceso-a-la-educacion-superior>. Acceso en: 17 nov. 2016.

GONZÁLEZ, Sara. Universidad y transiciones a la democracia en la Europa mediterránea e lberoamérica (1970-1980). Presentación. Espacio, Tiempo y Educación, v. 2, n. 2, p. 25-32.

HABERMAS, Jürgen. Historia y crítica de la opinión pública. La transformación estructural de la vida pública. Barcelona: Gustavo Gili, 1994.

HERNÁNDEZ HUERTA, José Luis; ORTEGA GAITE, Sonia. Civic Education and public opinion in Argentina during the transition to democracy (1982-1983). History of

Education \& Children's Literature, v. 10, n. 2, p. 359-389, 2015.

HERNÁNDEZ HUERTA, José Luis; GONZÁLEZ GÓMEZ, Sara. Opinión pública y educación durante la transición a la democracia en España. Primeras consideraciones y guía de fuentes documentales. Educació i Història: Revista d'Història de l'Educació, n. 24, p. 173-215, 2014.

KAUFMANN, C. (Dir.). Dictadura y Educación. Tomo 1: Universidad y Grupos Académicos Argentinos (1976-1983). 2 ed. Salamanca: FahrenHouse, 2017.

PIERELLA, María Paula. El ingreso a la universidad pública en la Argentina. Los profesores de primer año como actores claves en el diseño de políticas inclusivas. In: MARTÍNEZ, Silvia; CORREA, Néstor (Coords.). Desafíos y dilemas de la universidad y 
la ciencia en América Latina y el Caribe en el siglo XXI. Buenos Aires: Teseo, 2015.

RUIZ, Guillermo; CARDINAUX, Nancy (Comps.). La autonomía universitaria: definiciones normativas y jurisprudenciales en clave histórica y actual. Buenos Aires: La Ley / Facultad de Derecho de la Universidad de Buenos Aires, 2010.

RUIZ, María Consuelo; SCHOO, Susana. La obligatoriedad de la educación secundaria en América Latina. Convergencias y divergencias en cinco países. Foro de Educación, v. 16, n. 12, p. 71-98, 2014.

SIGAL, Víctor. El acceso a la Educación Superior. Serie Estudios y Propuestas. Argentina: Secretaría de Políticas Universitarias, Ministerio de Educación, 1995.

El sistema de admisión a la universidad en la Argentina. In: Sistemas de Admisión a la Universidad. Seminario Internacional. Argentina: Secretaría de Políticas Universitarias, Ministerio de Educación, 1999.

. La cuestión de la admisión a los estudios universitarios en Argentina. Documento de Trabajo n. 113, Universidad de Belgrano, 2003. Disponible en:

$<$ http://www.ub.edu.ar/investigaciones/dt_nuevos/113_sigal.pdf>. Acceso en: 29 sept. 2016.

TROMBETTA, Agustín. El ingreso en las universidades nacionales argentinas. In:

Sistemas de Admisión a la Universidad. Seminario Internacional. Argentina: Secretaría de Políticas Universitarias, Ministerio de Educación, 1999.

VOMMARO, Pablo. Juventudes y políticas en la Argentina y en América Latina. Tendencias, conflictos y desafíos. Buenos Aires: Grupo Editor Universitario, 2015.

\section{Fuentes documentales}

ABÁSOLO, Jorge. Aumento de las tensiones. Clarín, 8 dic. 1982.

. El avance de los estudiantes. Clarín, 27 oct. 1982.

. Los contrastes de la transición. Clarín, 16 mar. 1983.

. Tiempos de búsquedas. Clarín, 17 nov. 1982.

. Tropiezo estudiantil. Clarín, 23 mar. 1983.

. Vuelve el movimiento estudiantil. Clarín, 6 oct. 1982.

CHAPMAN, William. Sobre futuras reformas de la universidad estatal. La Nación, 27 jun. 1983.

DILLON, Alfredo. La Justicia bloqueó el ingreso irrestricto a la universidad. Clarín, 12 mayo 2016. Disponible en: <http://www.clarin.com/sociedad/Justicia-bloqueo-ingresoirrestricto-universidad_0_1574842707.html>. Acesso en: 7 nov. 2016.

GAINZA, Máximo. Ayuda a los soldados estudiantes. La Prensa, 25 jun. 1982.

. Demandas estudiantiles. La Prensa, 4 oct. 1983.

. Extraño petitorio estudiantil. La Prensa, 12 nov. 1982.

. Improcedentes protestas estudiantiles. La Prensa, 24 sept. 1983.

. Los soldados que regresan. La Prensa, 26 jun. 1982.

. Mal comienzo. La Prensa, 31 ago. 1982.

Reclamos estudiantiles. La Prensa, 16 mar. 1983.

HERRERA DE NOBLE, Ernestina. Las elecciones estudiantiles. Clarín, 14 jun. 1983. 
MITRE, Bartolomé. Cambios y riesgos. La Nación, 27 abr. 1983.

. Cuestionamientos. La Nación, 22 sept. 1982.

Derechos y deberes. La Nación, 30 jun. 1982.

Días difíciles. La Nación, 13 abr. 1983.

. El ingreso en la UBA. La Nación, 23 mar. 1983.

. El ingreso universitario. La Nación, 17 ago. 1983.

. Enseñar y aprender. La Nación, 20 may. 1983.

. Hacia la unidad estudiantil. La Nación, 16 nov. 1983.

. Los centros de estudiantes. La Nación, 6 abr. 1983.

. Participación estudiantil. La Nación, 20 abr. 1983.

. Rectores universitarios. La Nación, 2 nov. 1983.

. Testimonio de un aval. La Nación, 30 mar. 1983.

. Un hecho inédito. La Nación, 15 jun. 1983.

. Un marzo agitado La Nación, 16 mar. 1983.

. Un septiembre agitado. La Nación, 21 sept. 1983.

. Un síntoma significativo. La Nación, 18 may. 1983.

. Un toque de atención. La Nación, 16 feb. 1983.

. Universidad: la movilización estudiantil. La Nación, 19 ene. 1983.

SARA GONZÁLEZ GÓMEZ es Doctora en Ciencias de la Educación por la Universidad de Salamanca (Usal) y profesora del Departamento de Pedagogía y Didácticas específicas de la Universidad de las Islas Baleares (UIB). Es miembro del Grupo de Estudios de Historia de la Educación (Irie/UIB) y de los grupos Ágora de Educación (Universidad de Valladolid) y Memoria y proyecto de la educación (Usal). Es coeditora de las revistas Espacio, Tiempo y Educación y Foro de Educación y pertenece al consejo de redacción de Educació i Història. Endereço: Facultad de Educación - Carretera de Valldemossa, km. 7,5-07122 - Palma, Islas Baleares - España.

E-mail: sara.gonzalez@uib.es

GUILLERMO RAMÓN RUIZ es Doctor de la Universidad de Buenos Aires, área Ciencias de la Educación (UBA), Master of Arts in Education (University of California Los Angeles). Profesor catedrático en las Facultades de Derecho y de Psicología de la Universidad de Buenos Aires e Investigador del Consejo Nacional de Investigaciones Científicas y Técnicas (Conicet). Dirige proyectos de investigación en el marco de la programación científica UBACyT y de la Agencia Nacional de Promoción Científica y Tecnológica.

Endereço: Facultad de Derecho de la UBA - Avenida Figueroa Alcorta, 2263 - C1425CKB Ciudad de Buenos Aires - Argentina.

E-mail: gruiz@derecho.uba.ar 
Recebido em 01 de outubro de 2017.

Aceito em 28 de novembro de 2017.

(c) (i) 